\title{
Estudio sobre la retención y aplicación de los conceptos tratados en el Curso de Liderazgo Médico del Centro Médico ABC
}

\section{Study on the retention and application of the concepts discussed in the Medical Leadership Course of the ABC Medical Center}

\author{
José Octavio Ruiz Speare, ${ }^{\star}$ Denise Miranda Chávez, ${ }^{\ddagger}$ Francisco Valdés Saldaña, ${ }^{\S}$ \\ María Elena Manzanaque," Blanca Velázquez Hernández
}

\begin{abstract}
RESUMEN
Introducción: El cuidado de la salud en todo el mundo provoca una desconexión persistente y cada vez más acentuada entre la capacidad de la fuerza de trabajo de liderazgo médico y las necesidades de nuestros sistemas de salud cada vez más complejas. Cerrar esta brecha requiere habilidades de liderazgo que no se adquieren durante la capacitación médica tradicional. Objetivo: Establecer la retención y aplicación de los conceptos tratados en el Curso de Liderazgo Médico del Centro Médico ABC. Material y métodos: Estudio descriptivo, observacional, retrospectivo y transversal. Encuesta con 25 preguntas realizada a 253 médicos que participaron en 11 cursos de liderazgo médico entre 2013 y 2018 para evaluar la retención y aplicación de conceptos tratados en los mismos. Contestaron 136 médicos (54\%). Resultados: $100 \%$ de los médicos recuerda las habilidades de liderazgo enseñadas en el curso; $97 \%$ considera que los resultados al poner en práctica las habilidades de liderazgo aprendidas han sido efectivas. Todos refieren que el curso ha facilitado su trabajo institucional. El 90\% desconocía muchas de las actividades que se llevan a cabo en el Centro Médico ABC. El 98\% de los alumnos participantes recomendarían el curso y $90 \%$ lo volvería a tomar. La creación de líderes clínicos se traducirá en mejores resultados clínicos, beneficio de los pacientes y gran
\end{abstract}

\begin{abstract}
Introduction: Health care worldwide causes a persistent and increasingly worse disconnect between the ability of the medical leadership workforce and the needs of our increasingly complex health systems. Closing this gap requires leadership skills that are not acquired during traditional medical training. Objective: Establish the retention and application of the concepts discussed in the Medical Leadership Course of the ABC Medical Center. Material and methods: Survey with 25 questions made to 253 doctors who participated in 11 medical leadership courses between 2013 and 2018 to evaluate retention and application of concepts treated in the course. Results: $100 \%$ of doctors remember the leadership skills taught in the course, $97 \%$ believe that the results in putting into practice the leadership skills learned have been effective. $100 \%$ report that the course has facilitated their institutional work. 90\% of doctors were unaware of many of the activities carried out at the ABC Medical Center. $98 \%$ of participating students would recommend the course and $90 \%$ would take it again. The creation of clinical leaders will result in better clinical outcomes, benefit of our patients and great learning for all. The results obtained in the present study are a great stimulus to continue our Medical Leadership Program
\end{abstract}

* Director del Centro de Educación Médica por Simuladores y Estudios en liderazgo Médico.

¥ Psicóloga. Jefe de Educación de las Clínicas de Impacto Social.

$\S$ Técnico en Urgencias Médicas. Coordinador del Centro de Educación Médica por Simuladores.

"Médico cirujano, servicio social Centro Médico ABC.

१ Directora Corporativa de las Clínicas de Impacto Social.

Centro Médico ABC.

Recibido para publicación: 15/10/2019. Aceptado: 04/03/2020.

\section{Correspondencia:}

José Octavio Ruiz Speare

Avenida Paseo de las Palmas Núm. 745-901,

Lomas de Chapultepec,

Alcaldía Miguel Hidalgo, 11000,

Ciudad de México.

Tel: 555520 5451; Cel: 5543691991

E-mail: octavio.ruiz41@gmail.com

Abreviatura:

$\mathrm{CMABC}=$ Centro Médico ABC. 
aprendizaje para todos. Los conceptos tratados alcanzaron un alto grado de retención, aplicación y satisfacción. Los resultados obtenidos en el presente estudio son un gran estímulo para continuar nuestro programa de liderazgo médico; en especial para enseñarlo y evaluarlo. Conclusión: El liderazgo clínico efectivo debe enseñarse y practicarse en las instituciones de salud.

Palabras clave: Retención, aplicación, liderazgo, autoeficacia. Nivel de evidencia: IV

\section{INTRODUCCIÓN}

Desde la aparición del hombre en la Tierra, han existido los líderes. En la familia, el clan, la tribu, en el desarrollo de cada pueblo, ciudad o país, y en todo tipo de instituciones, la figura del líder ha estado presente. En toda actividad humana, política, militar, cultural, artística, musical, industrial, de investigación y, desde luego, en la medicina, el liderazgo ha sido fundamental para promover el cambio y el progreso a través de los siglos. ${ }^{1,2}$

Para su estudio y enseñanza se han publicado libros, desarrollado cursos y talleres, licenciaturas e, incluso, doctorados en liderazgo. Una búsqueda rápida en Amazon revela que existen más de 120,000 libros sobre el tema. ${ }^{3}$

Dentro de estos aspectos se ha descrito un gran número de definiciones de liderazgo, y revisiones muestran que existen más de 100 descripciones del concepto. ${ }^{4-7}$ De entre todas ellas, nos permitimos señalar tres que consideramos las más explícitas:

"Liderazgo es un proceso mediante el cual un individuo ejerce influencia sobre un grupo de individuos para lograr un objetivo común.» ${ }^{4}$

Northouse $\mathrm{P}$

«El liderazgo es influencia.» 6 John C Maxwell

«Liderazgo es la actitud que mueve a la iniciativa de un individuo para cambiar el estado de las cosas que deben ser cambiadas.» ${ }^{7}$ José Luis Taddei

El cuidado de la salud en todo el mundo provoca una desconexión persistente y cada vez peor entre la capacidad de la fuerza de trabajo de liderazgo médico y las necesidades de nuestros sistemas de salud cada vez más complejos. Cerrar esta brecha requiere habilidades de liderazgo que no se adquieren durante la capacitación médica tradicional. ${ }^{8-10}$ especially in teaching and evaluating it. Conclusion: Effective clinical leadership must be taught and practiced in health institutions.

Keywords: Retention, application, leadership, self-efficacy.

Level of evidence: $I V$

Ante este panorama, el reto de la atención médica actual es un problema complejo, la pirámide poblacional con gente cada vez de mayor edad y presencia de las enfermedades crónico-degenerativas que van de la mano con el envejecimiento, provocan una continua elevación en los costos del cuidado de la salud. Esto significa una necesidad urgente en la creación de modelos efectivos para el cuidado de la salud, con un manejo adecuado de las operaciones a nivel local. Los médicos clínicos tienen una gran influencia en ambos aspectos, la asistencial y el cuidar los recursos. ${ }^{11}$

Promovido por la Dirección del Cuerpo Médico del Centro Médico ABC (CMABC), en el año 2010, se realizó un estudio de planeación estratégica en la que se determinaron las fortalezas y debilidades del mismo; se propusieron medidas para resolver sus debilidades. ${ }^{12}$ Se planteó la necesidad de crear una cultura de liderazgo a través de un programa que tuviese como objetivo crear líderes clínicos y facilitar la visión, tanto del cuerpo médico como de la institución. La Dirección General y la Dirección del Cuerpo Médico en conjunto diseñaron un curso de Liderazgo Médico, con la finalidad fundamental de identificar individuos con capacidad de liderazgo y el desarrollo de conocimientos, habilidades y actitudes de liderazgo en los miembros del cuerpo médico del CMABC. Esta planificación fue y es congruente ante los retos que implica el futuro en la atención de la salud, expuesto en la parte inicial de esta introducción.

En septiembre del 2013, se realizó el Primer Curso de Liderazgo Médico, impartido por profesores con experiencia en cursos de educación y liderazgo. ${ }^{13}$ Desde entonces se han realizado 12 cursos en los que han participado 253 alumnos, con duración de un día y medio, se obtuvieron 14 horas de crédito de educación médica continua, otorgadas por el American College of Surgeons (capítulo México). El curso es totalmente interactivo, las conferencias son dictadas por los mismos profesores, quienes han adquirido la maestría en su conferencia respectiva. 


\section{Profesores del Curso de Liderazgo Médico en el CMABC:}

- CP. Alejandro Alfonso Díaz, Director General del CMABC.

- Dr. Elías Horta Bustillo, Vicepresidente Médico.

- Dra. Blanca Velázquez Hernández, Directora Corporativa Clínicas de Impacto Social.

- Dra. Ana Luisa Argomedo Manrique, Gastroenteróloga y Endoscopista. National Educator ACS.

- Dra. Elisa Bustamante García, Vicepresidencia Médica.

- Dr. Marino Capurso García FACS, Cirujano General.

- Dr. Vicente Cardona Infante FACS, Cirujano General.

- Dr. Moisés Zielanowski Mansbach FACS, Cirujano General.

- Dr. José Guadalupe Arizmendi Dorantes, MSc en Educación Pediatra Neonatólogo.

- Dr. Armando Torres Gómez FACS, Cirujano Ortopedista.

- Maestra Paulina Trápaga Sánchez, Jefe de Investigación CMABC.

- Dra. Adriana Hernández FACS, Cirujano General.

- Dra. Estefania Villalobos Rubalcava FACS, Cirujano General.

- Dr. Juan Felipe Sánchez Marle FACS, Cirujano de Cabeza y Cuello.

- Maestra Yessica Sánchez Romero

- Dr. José Octavio Ruiz Speare FACS, Cirujano General. National Educator ACS.

Se considera parte medular del curso el trabajo en mesas de discusión correspondientes a cada una de las conferencias. En todo momento se evalúan conocimientos, habilidades y actitudes. En la Tabla 1 se muestra el programa del Curso de Liderazgo.

\section{MATERIAL Y MÉTODOS}

Estudio descriptivo, observacional, retrospectivo y transversal.

En los primeros 11 cursos de Liderazgo Médico (2013-2018) participaron 253 médicos, a los cuales se envió un cuestionario (Anexo 1) con 25 preguntas, mediante los Formularios de Google; 136 (54\%) lo contestaron.

\section{RESULTADOS}

De los 136 médicos, el mayor número de respuestas se obtuvo de los alumnos que tomaron el curso entre el 2015 y 2016.
Tabla 1: Temas tratados en el curso de Liderazgo Médico.

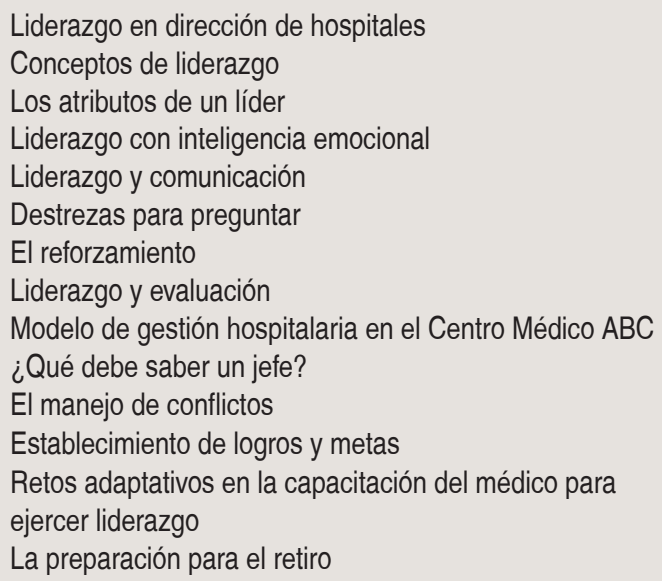

Un $33 \%$ de los alumnos fueron del sexo femenino y $82 \%$ del masculino. De los médicos que contestaron, $57 \%$ fueron mujeres y $43 \%$ fueron hombres.

El 70\% de los alumnos que participaron en el curso se encuentra entre los 30 y 50 años (etapa de despegue y de mayor producción clínica y académica). El porcentaje entre los 50 y 60 años es semejante; en este grupo se notó, además de su experiencia clínica, que se encuentran bien establecidos en su práctica médica y la mayoría tienen actividades académicas, pero sus habilidades de liderazgo no fueron mejores que las de los médicos jóvenes. El 80\% de los participantes en el curso realizan sus actividades en el CMABC.

El 100\% de los alumnos refiere recordar las habilidades de liderazgo enseñadas en el curso, y de ellos $64 \%$ las recuerda muy bien.

El $70 \%$ ha aplicado todas las habilidades de liderazgo sugeridas en el curso, $90 \%$ ha puesto en marcha «el saber escuchar», uno de los objetivos más importantes del curso (Figura 1).

El 97\% de los alumnos considera que los resultados de poner en práctica las habilidades de liderazgo aprendidas en el curso han sido satisfactorios. El 83\% de los alumnos cumplen con los objetivos del curso: saber escuchar y saber preguntar.

El reforzamiento es el tema más controvertido del curso y el más difícil de entender, practicar e implementar. El 65\% de los alumnos lo entiende, recuerda y practica. La mitad de los alumnos que en esta pregunta contestaron «no», señala que no ha tenido oportunidad de practicarlo. Una minoría señala que no entendió el concepto. A pesar de que el tema es relativamente nuevo, $100 \%$ lo comprendió, $83 \%$ lo aplica y $17 \%$ lo aplica ocasionalmente. El tema fue de su agrado. 
Las habilidades para manejar las emociones fueron entendidas y prácticamente $100 \%$ son aplicadas en todos los aspectos de la vida. La mitad de los encuestados refiere aplicar los conocimientos adquiridos en forma muy satisfactoria (Figura 2). El 70\% de los alumnos dan una importancia semejante, tanto a las conferencias interactivas como a las mesas de trabajo. Un porcentaje elevado (por arriba de 80\%) refiere que el curso tuvo un efecto positivo en todos los aspectos de su vida (Figura 3). La percepción que tienen con respecto a cómo los ven sus colaboradores es excelente en $100 \%$, lo que refleja una elevada autoestima, hay motivación y posiblemente un buen reforzamiento en ambas direcciones. Más de $80 \%$ refieren que su trabajo se ha facilitado y mantienen

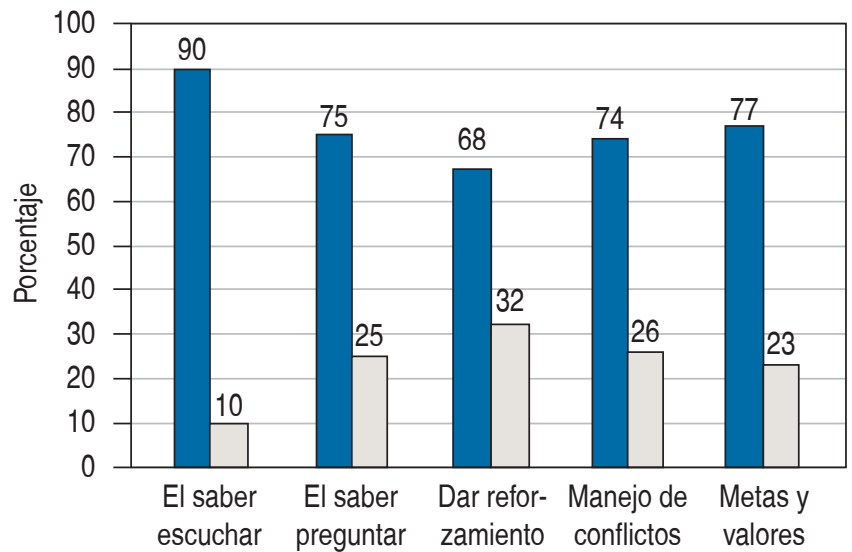

$\square$ Sí $\square$ Algunas veces $\square$ Nunca

Figura 1: ¿Has puesto en práctica estas habilidades?

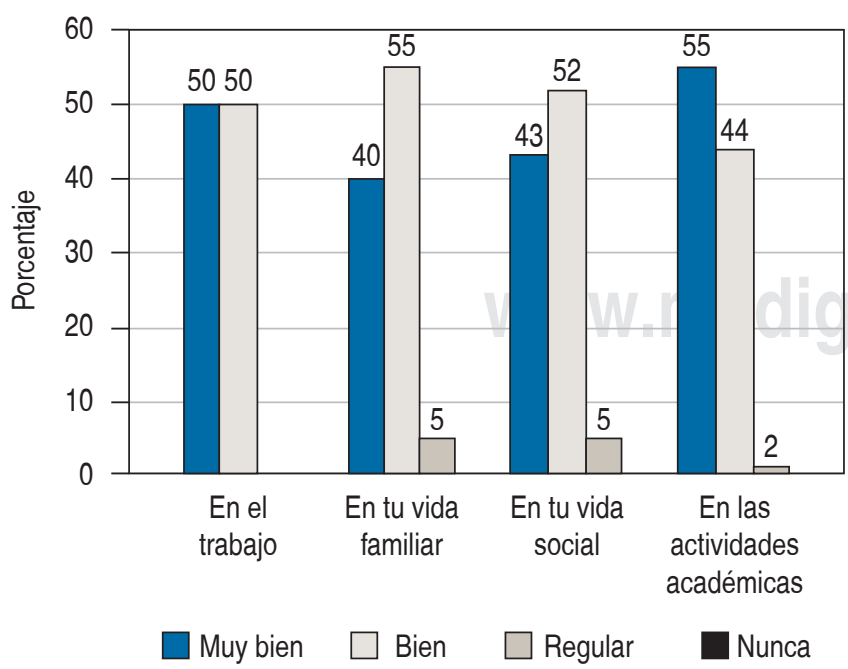

Figura 2: ¿Cómo evaluarías tus habilidades para regular tus emociones?

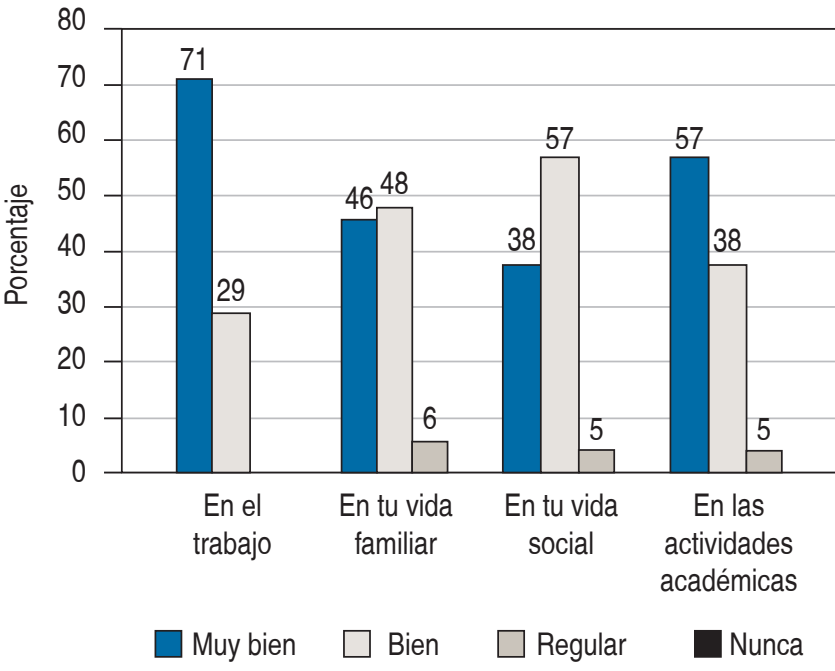

Figura 3: ¿El curso fue de utilidad para mejorar tu desarrollo en tu trabajo, en tu vida familiar, en tu vida social, en tus actividades académicas?

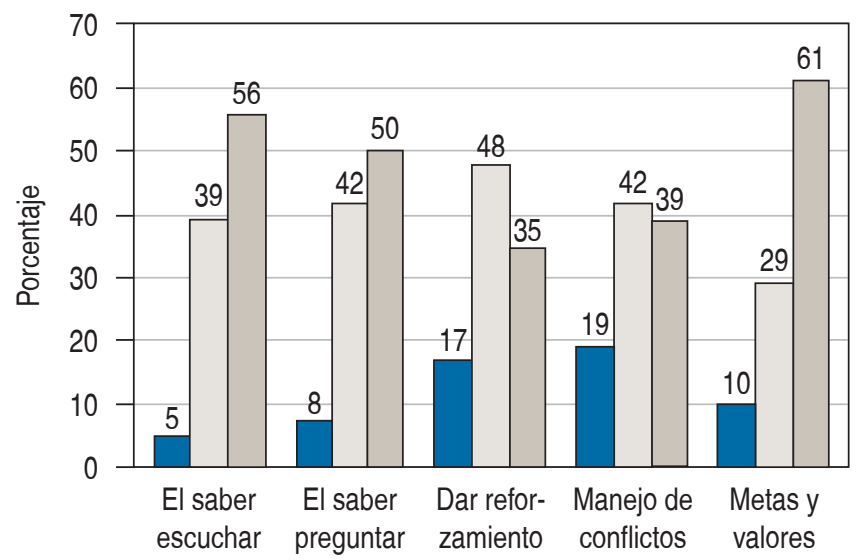

Falta desarrollarlo $\square$ Algo desarrollado $\square$ Está desarrollado

Figura 4: ¿Cuáles son las habilidades de liderazgo que consideras que te hace falta desarrollar?

mejores relaciones con sus colaboradores. El 97\% de los alumnos perciben que sus jefes los ven bien, lo que refleja satisfacción en su trabajo y elevada autoestima. El $91 \%$ de los alumnos se perciben con habilidades de liderazgo, lo que significa que entienden el concepto, están desarrollándose en un medio que les ofrece ejercer los principios de liderazgo, les gusta y refleja el desarrollo de una cultura institucional de liderazgo. En la Figura 4 se muestra que 50\% percibe que conoce y ha desarrollado en forma adecuada sus habilidades de liderazgo señaladas. Dos terceras partes tienen claro el concepto de metas y valores. Como era de esperarse por el grado de dificultad en 
aprenderlo y aplicarlo, el reforzamiento es la habilidad menos desarrollada.

Noventa por ciento de los miembros del cuerpo médico desconocían muchas de las actividades que se desarrollan en la institución en donde realizan su práctica médica. Este hallazgo es el de mayor importancia. Es necesario promover reuniones que estimulen este conocimiento hacia el cuerpo médico. Se mejoró la comunicación en un porcentaje importante, en especial con los demás compañeros del cuerpo médico y el personal administrativo (Figura 5). La tercera parte de los alumnos ha logrado un ascenso o promoción en su trabajo. Aunque esto se logra no necesariamente por el curso, éste ha facilitado su trabajo en su desarrollo laboral, tanto para sus colaboradores como para sus superiores.

De acuerdo con las respuestas, los conceptos planteados en el curso son útiles en todas las etapas de la vida. El 50\% de los alumnos se encuentra por arriba de 40 años y tienen alrededor de 10 o 15 años de práctica establecida. El mayor porcentaje (56\%) está en los médicos de práctica médica ya establecida, cuya visión es más clara al ser de mayor interés y utilidad el poder desarrollar sus habilidades de liderazgo en esa etapa de la vida.

El 98\% de los alumnos recomendaría a sus amigos o colaboradores tomar el curso. Esta respuesta habla de la calidad y utilidad de éste y es el mejor reforzamiento para los Profesores del Curso.

El 89\% de los alumnos volvería a tomarlo. Esta respuesta indica que se cumplieron los objetivos de la enseñanza en el adulto, sus respuestas han señalado que aprendieron. Seguramente volverían a tomar el curso, porque se divirtieron. Es como volver a ver una película que te gustó.

\section{DISCUSIÓN}

Tradicionalmente, los médicos se seleccionan con responsabilidades de liderazgo en función de su prestigio nacional como maestros clínicos de excelencia, investigadores destacados y/o educadores venerados. Estas credenciales siguen siendo importantes, pero no son suficientes en el clima actual en la atención a la salud. Dada al constante cambio en nombramientos entre los médicos líderes, como jefes de departamento y decanos, ya no podemos permitirnos descuidar las habilidades que son esenciales para que los líderes tengan éxito.

Creemos que existe la necesidad de una nueva generación de guías que puedan promover la alineación estratégica y cultural ante un cambio rápido.

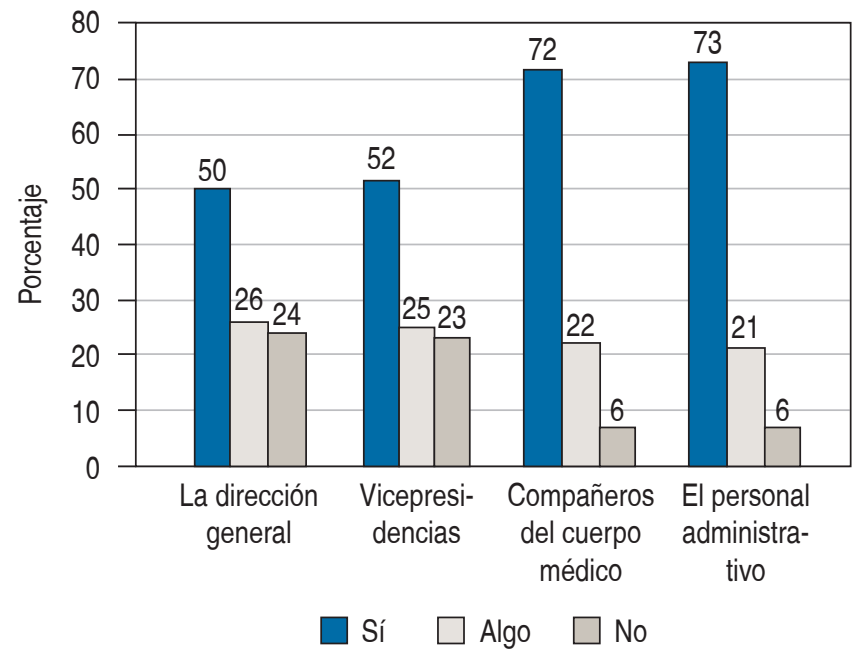

Figura 5: ¿Después del curso mejoró tu comunicación?

Apoyar el crecimiento de tales líderes requiere tiempo y compromiso. El desarrollo del liderazgo debe ser durante la escuela de medicina y los líderes potenciales pueden nutrirse en cada etapa del avance profesional. Sugerimos que los sistemas de salud se centren en estrategias para promover el desarrollo efectivo de los líderes médicos..$^{1,2,8}$ Toda institución de salud debe incluir en su visión la formación de líderes clínicos.

Si dirigimos la atención a la Visión del Cuerpo Médico del CMABC redactada en 2011, se señala claramente la palabra liderazgo, y a la letra dice:

Cuerpo Médico del Centro Médico ABC que a través de un liderazgo efectivo y visionario en los campos de la asistencia, investigación y enseñanza, promoviendo y mejorando constantemente sus conocimientos, habilidades y actitudes en forma individual y colectiva apoyándose en tecnología de punta, será reconocido por su desarrollo de excelencia.

El liderazgo reviste tal importancia que está en las declaraciones de la misión, los valores y la visión de muchas instituciones de salud y educativas; es indispensable incluir en los métodos formativos, procesos educativos que promuevan la adquisición de habilidades de liderazgo. Se une a la visión de la institución y se establece la necesidad de formar líderes clínicos y es fundamental enseñar liderazgo y, sobre todo, evaluarlo.

En cuanto a las experiencias de programas educativos sobre liderazgo en medicina, existen abundantes publicaciones y cursos. ${ }^{3,14-17}$ Las conclusiones de 
An Med (Mex) 2020; 65 (1): 27-34

estos estudios son que existe la necesidad de desarrollar competencias de liderazgo. ${ }^{15}$

Straus y colaboradores ${ }^{17}$ encontraron que la educación en liderazgo y participación en cursos del mismo incrementó en forma significativa el rango académico (21 a 48\%), su posición de liderazgo en el hospital del 9 al 30\% y aumentaron sus publicaciones de 2.1 a 3.5 por año.

El presente estudio es un esfuerzo para incrementar la cultura de liderazgo en nuestra institución y en la medicina mexicana. Los resultados obtenidos en los médicos que han participado muestran un alto grado de satisfacción, con un elevado porcentaje en la retención y aplicación de las habilidades de liderazgo aprendidas, y de acuerdo con sus respuestas, el mejor reforzamiento que pueden obtener los profesores es que prácticamente $100 \%$ recomendaría el curso y $90 \%$ lo volvería a tomar.

Evidentemente, en las respuestas obtenidas se observa el fenómeno de autoeficacia. Ésta significa que el conocimiento que los individuos tienen acerca de sus capacidades les da la confianza para alcanzar una meta o enfrentar una nueva situación. Es un concepto en educación que nos habla de qué tan capaz se siente el alumno para aplicar sus habilidades y aprender nuevas. Logra creer que él puede y acepta nuevos retos. ${ }^{17}$

El presente trabajo colabora con una evidencia científica que apoya la educación en liderazgo y un cambio organizacional en las ciencias de la salud. Un liderazgo efectivo, como está señalado en nuestra visión, se traducirá en mejores resultados clínicos, beneficio de nuestros pacientes y gran aprendizaje para todos. Los resultados obtenidos en el presente estudio son un gran estímulo para continuar nuestro Programa de Liderazgo Médico, promoverlo, enseñarlo y evaluarlo.

\section{CONCLUSIÓN}

La creación de líderes médicos se traducirá en mejores resultados clínicos, y un importante beneficio en la calidad de la atención de nuestros pacientes. Ésta es una de las mejores habilidades para enfrentar los retos médicos, tecnológicos, económicos y académicos frente a los cambios en la medicina del siglo XXI. Los resultados obtenidos en el presente estudio son un gran estímulo para continuar nuestro Programa de
Liderazgo Médico, en especial para enseñarlo y evaluarlo. El liderazgo clínico efectivo debe enseñarse y practicarse en las instituciones de salud.

\section{AGRADECIMIENTOS}

El más profundo agradecimiento a los profesores del Curso de Liderazgo Médico del CMABC, cuyos valores de lealtad, entusiasmo, profesionalismo, dedicación y ejemplo han hecho posible la realización y continuidad de este curso.

\section{BIBLIOGRAFÍA}

1. Ruiz JO. Liderazgo en medicina. An Med (Mex). 2014; 59 (3): 219227.

2. Ruiz JO. Liderazgo. Capítulo 1 ¿Qué es liderazgo? Editorial Alfil. México, 2017.

3. Sánchez M. Liderazgo en medicina: ¿debemos enseñarlo y evaluarlo? Inv Ed Med. 2015; 4 (14): 99-107.

4. Northouse P. Leadership theory and practice. $8^{\text {th }}$ ed., Western Michigan University. SAGE Publications, 2018.

5. Rost JC. Leadership for the twenty-first century. Wesport, CO: Praeger, 1991.

6. Maxwell JC. Developing the leader within you: developing the leaders around you. Nashville, TN: Thomas Nelson, 1993.

7. Taddei JL. [Consultada 15/03/19] Disponible en: www. sigueme.net/liderazgo/.

8. Lerman C, Jameson JL. Leadership development in medicine. N Engl J Med. 2018; 20: 1862-1863.

9. Dzau VJ, Cho A, Ellaissi W, Yoediono Z, Sangvai D, Shah B, et al. Transforming academic health centers for an uncertain future. N Engl J Med. 2013; 369 (11): 991-993.

10. Ramsey PG, Miller ED. A single mission for academic medicine: improving health. JAMA. 2009; 301: 1475-1476.

11. Bohmer MJ. Leading clinicians and clinicians leading. N Engl J Med. 2013; 368: 1468-1470.

12. Ruiz SJ, Hurtado C. Estudio de Planeación Estratégica tipo FODA en el Cuerpo Médico del American British Cowdray Medical Center. An Med (Mex). 2011; 56 (4): 173-183.

13. Colegio Americano de Cirujanos Capítulo México, Curso "Educación y Liderazgo para Médicos". [Consultada 15/03/19 http://atls.mx/cursos educación-liderazgo.htm/.

14. Souba WW, Day DV. Leadership values in academic medicine. Acad Med. 2006; 81:20-6.

15. Abbas MR, Quince TA, Wood DF, Benson JA. Attitudes of medical students to medical leadership and management: a systematic review to inform curriculum. Development. BMC Med Educ. 2011; 11: 93.

16. Straus SE, Soobiah C, Levinson W. The impact of leadership training programs on physicians in academic medical centers: a systematic review. Acad Med. 2013; 88 (5): 710-723.

17. [Consultada (04/09/19] Disponible en: https://www.significados. com > autoeficacia. 


\section{Anexo 1: Cuestionario aplicado para el estudio sobre la retención y aplicación de los conceptos tratados en el Curso de Liderazgo Médico del Centro Médico ABC del 2013 al 2018.}

1. Participé en el Curso de Liderazgo Médico en el año de:
( ) 2013
( ) 2014
( ) 2015
( ) 2016
( ) 2017
( ) 2018
( ) Otra

2. Indique su género:
( ) Mujer
( ) Hombre

3. ¿Qué edad tienes?
( ) Entre 30 y 40 años
( ) Entre 40 y 50 años
( ) Entre 50 y 60 años
( ) Más de 60 años
( ) Otra

4. ¿Te encuentras realizando tus actividades en el Centro Médico ABC?
( ) Sí
( ) No

5. ¿Recuerdas las habilidades de liderazgo enseñadas en el curso?

( ) Todas muy bien

( ) Alguna

( ) Ninguna

6. ¿Has puesto en práctica estas habilidades?

\section{Sí Algunas veces Nunca}

$\begin{array}{llll}\text { El saber preguntar } & (\text { ) } & \text { ( ) } & \text { ( ) } \\ \text { Dar reforzamiento } & (\text { ) } & \text { ( ) } & \text { ( ) } \\ \text { El saber escuchar } & (\text { ) } & (\text { ) } & (\text { ) } \\ \text { Manejo los conflictos } & (\text { ) } & (\text { ) } & (\text { ) } \\ \text { Metas y valores } & \text { ( ) } & \text { ( ) } & \text { ( ) }\end{array}$

7. En general, ¿nos puedes decir si los resultados de poner en práctica estas habilidades han sido...?

( ) Buenos

( ) Regulares

( ) Sin resultados

8. Desde que tomaste el curso ¿escuchas y preguntas con mayor frecuencia?

( ) Sí

( ) Algunas veces

( ) No

9. ¿Has logrado practicar y dar el reforzamiento?

( ) Sí

( ) Algunas veces

( ) No

10. Si la respuesta a la anterior es no:

( ) Porque no entendí el concepto

( ) Porque se me olvidó

( ) Porque no tengo oportunidad de practicarlo

( ) Porque no creo que sirva

11. ¿Has puesto en práctica el concepto de inteligencia emocional?

( ) Sí

( ) Algunas veces

( ) No

12. ¿Cómo evaluarías tus habilidades para regular tus emociones?

En tus actividades ( ) ( ) ( ) ( )

13. ¿En qué parte del curso consideras que la enseñanza tiene mayor efectividad?

( ) Las conferencias

( ) Las mesas de trabajo

( ) Ambas por igual 
14. El curso fue de utilidad para un mejor desarrollo en:

$\begin{array}{lcccc} & \text { Muy Bien } & \text { Bien } & \text { Regular } & \text { Mal } \\ \text { En el trabajo } & (\text { ) } & (\text { ) } & (\text { ) } & (\text { ) } \\ \text { En tu vida familiar } & (\text { ) } & (\text { ) } & (\text { ) } & (\text { ) } \\ \text { En tu vida social } & (\text { ) } & (\text { ) } & (\text { ) } & (\text { ) } \\ \text { En tus actividades } & & & & \\ \text { académicas } & (\text { ) } & (\text { ) } & (\text { ) } & (\text { ) }\end{array}$

15. ¿Crees que tus colaboradores te perciben como un elemento competente?
( ) Muy bien
( ) Bien
( ) Regular
( ) Mal

16. ¿Percibes que después de tomar el curso se facilitó tu trabajo y hay mejores relaciones con tus colaboradores?
( ) Sí
( ) Algo
( ) No

17.¿Cómo sientes que te perciben tus superiores?
( ) Muy bien
( ) Bien
( ) Regular
( ) Mal

18. ¿Cuál es tu percepción de tus habilidades de liderazgo?
( ) Buena
( ) Regular
( ) Mala

19. ¿Qué habilidades de liderazgo consideras que te hace falta desarrollar?

1. El saber escuchar

2. El saber preguntar

3. Dar reforzamiento

4. Manejo de los conflictos

5. Metas y valores
( ) Falta desarrollarlo

( ) Algo desarrollado

( ) Está desarrollado

20. ¿A través del curso conociste un mayor número de actividades que se realizan en el Centro Médico ABC?
( ) Sí
( ) No

21. ¿Después del curso mejoró tu comunicación con?

$\begin{array}{lccc} & \text { Sí } & \text { Algo } & \text { No } \\ \text { La Dirección General } & (\text { ) } & (\text { ) } & (\text { ) } \\ \text { Vicepresidencias } & (\text { ) } & (\text { ) } & (\text { ) } \\ \text { Dar reforzamiento } & (\text { ) } & (\text { ( ) } & (\text { ( ) } \\ \text { Compañeros del Cuerpo Médico } & (\text { ) } & (\text { ) } & (\text { ) } \\ \text { El personal Administrativo } & (\text { ) } & (\text { ) } & (\text { ) }\end{array}$

22. Desde que tomaste el curso iha ocurrido un ascenso o promoción en tu trabajo?
( ) Sí
( ) No

23. ¿En qué etapa de tu vida profesional consideras que los conceptos planteados en el curso pueden ser de mayor utilidad?

( ) Durante la residencia

( ) En la etapa inicial de la práctica médica

( ) Como médico ya establecido y con una visión clara de tu futuro

24. ¿Recomendarías el curso?
( ) Sí
( ) Me es indiferente
( ) No

25. ¿Volverías a tomar el curso?
( ) Sí
( ) Me es indiferente
( ) No 